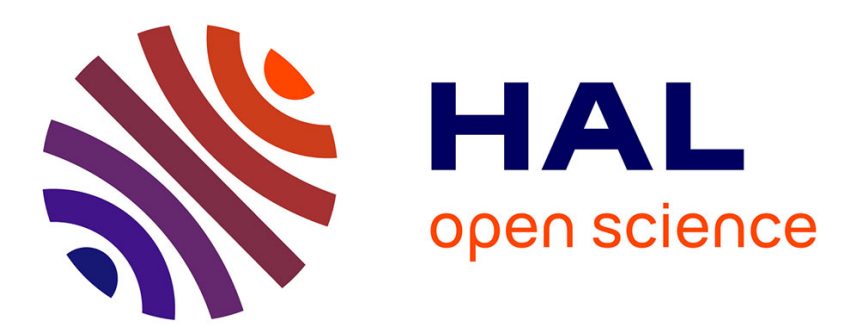

\title{
Les réseaux personnels des doctorants dans des chaînes latentes - Retour sur une recherche-action
}

\author{
Marie-Pierre Renée Bes, Nathalie Chauvac
}

\section{To cite this version:}

Marie-Pierre Renée Bes, Nathalie Chauvac. Les réseaux personnels des doctorants dans des chaînes latentes - Retour sur une recherche-action. Bulletin de Méthodologie Sociologique / Bulletin of Sociological Methodology, 2014, 121 (1), pp.59-74. 10.1177/0759106313509929 . hal-01519639

\section{HAL Id: hal-01519639 \\ https://hal-univ-tlse2.archives-ouvertes.fr/hal-01519639}

Submitted on 8 May 2017

HAL is a multi-disciplinary open access archive for the deposit and dissemination of scientific research documents, whether they are published or not. The documents may come from teaching and research institutions in France or abroad, or from public or private research centers.
L'archive ouverte pluridisciplinaire HAL, est destinée au dépôt et à la diffusion de documents scientifiques de niveau recherche, publiés ou non, émanant des établissements d'enseignement et de recherche français ou étrangers, des laboratoires publics ou privés. 
Les réseaux personnels des doctorants dans des chaînes latentes : retour sur une recherche-action

Marie-Pierre Bès ${ }^{1}$ (LISST \& ISAE, Université de Toulouse) et Nathalie Chauvac (LISST, Université de Toulouse)

\section{Introduction : des relations dans des chaines}

Les travaux sur les réseaux personnels portent, soit sur la compréhension des « entourages » et des sociabilités individuelles en mettant en évidence la taille des réseaux, la qualification des liens, l'interconnaissance ou non des membres, etc., soit sur la mise en évidence du capital social, c'est-à-dire de la capacité des acteurs à mobiliser leurs alter pour l'accès à des ressources (informations, objets, relations, etc.) ou pour des enjeux précis (emploi, affaires, par exemple). Les méthodologies employées (générateurs de noms, générateurs de positions) permettent surtout de mettre en évidence le versant «visible et positif» des réseaux, à savoir des relations effectives, agréables, choisies, activées, mobilisables. Or, dès que l'on adopte une approche processuelle des relations, force est de constater que l'on trouve alors des séries de relations moins positives, passant parfois par des liens faibles (Granovetter 1974 ), qui dépassent à la fois les réseaux personnels isolés et les réseaux complets aux frontières poreuses. Cela nécessite de tenir compte des relations qui existent entre les réseaux personnels, qui transcendent les cercles sociaux, et de chercher à faire émerger des chaînes relationnelles. C'est l'angle d'attaque des travaux sur les petits mondes, qui font suite à l'expérience de Milgram où apparaissent plus clairement des relations moins

$1 \quad$ Auteur pour la correspondance : bes@univ-tlse2.fr 
fortes, éloignées, parfois oubliées, mais également des barrières infranchissables, comme dans le cas d'obstacles raciaux, professionnels ou géographiques.

Notre étude part précisément de ces deux points de vue : d'une part, étudier des chaînes de relations, constituées en partie de différents réseaux personnels et d'autre part, mettre en évidence les points de blocage d'activation des liens, dans une situation de réseaux hétérophiles. A cet effet, l'opportunité de travailler sur les réseaux des doctorants, eu égard à leurs entourages professionnels hiérarchiquement et institutionnellement plus élevés, nous permettait de répondre à un certain nombre de questions posées autour de la thématique générale des « autres » liens ${ }^{2}$.

La problématique a tourné autour de deux grandes questions :

- en quoi la prise de conscience de son propre réseau et du réseau de ses proches permet-elle de mieux comprendre les processus relationnels en cours dans une activité précise ? A quoi peut servir le travail de révélation de son réseau et de celui de ses proches collègues?

- quelles chaînes relationnelles un individu imagine-t-il lorsqu'il souhaite accéder à une ressource ? Active-t-il ces chaînes ? Comment le fait-il ? Passe-t-il par son réseau personnel? par celui de ses proches? Lorsqu'il ne le fait pas ou n'envisage pas de le faire, quelles explications peut-on en donner?

Les réponses apportées dans cet article sur le cas spécifique des doctorants qui ont

2 Ce texte est issu d'une présentation à la 7ème journée ReSTo et 3e journée InterCongrès RT26 « Liens négatifs, liens perdus, liens latents » des 5 \& 6 avril 2012 à l'Université Toulouse le Mirail. Merci aux participants pour leurs remarques encourageantes et constructives. 
accepté de suivre notre formation en vue de trouver des financements de thèse ou des ressources, nous permettent en conclusion, de proposer une définition générique de la notion de chaîne latente, applicable à d'autres situations.

\section{Les doctorants ${ }^{3}:$ relations existantes et ressources attendues}

S'agissant des doctorants, nous partons de deux éléments de contexte : d'un côté, leur devenir après le doctorat dépend en grande partie des relations tissées pendant la thèse soit dans le milieu académique, soit vers le monde socio-économique (Godechot, \& Louvet, 2010 ; Shinn, 1998 ; Bès, 2004). Ainsi, certains bénéficient très tôt de la mise à disposition du réseau personnel du directeur de thèse tandis que d'autres sont moins soutenus dans l'accès aux ressources. D'un autre côté, il est admis que les façons de conduire les thèses diffèrent selon les « habitus » disciplinaires : il existe, grosso modo, deux modèles opposés : celui des sciences expérimentales où les chercheurs sont présents dans leurs laboratoires et conduisent des recherches essentiellement collectives à l'opposé de celui des Sciences Humaines (par exemple, l'anthropologie) où les chercheurs travaillent plus souvent seuls et dans différents lieux. Au niveau des jeunes chercheurs, ces « habitus » de travail induiraient aussi pour eux des différences d'accès aux ressources. Ainsi, certains doctorants seraient trop isolés pendant leurs thèses tandis que d'autres multiplieraient les atouts y compris relationnels (financements, réseau, participation à des projets ou à des séminaires, insertion dans les laboratoires, etc.). C'est précisément le lien entre la conduite de la thèse et les relations professionnelles du doctorant que nous souhaiterions interroger ici avec l'hypothèse sous-jacente que les

3 Pour alléger la lecture, nous choisissons le terme de doctorants, alors qu'il désigne aussi les doctorantes. Il en est de même pour les Directeurs et Directrices de thèse. 
manières de travailler du doctorant dépendent fortement, à la fois de la conscience et de la connaissance qu'il a du réseau professionnel qui l'entoure, et, également des occasions d'activer ce réseau potentiel (Bès \& Chauvac 2011). L'idée est donc de comprendre la nature et la structure des réseaux professionnels des doctorants tels qu'ils se les représentent et de montrer l'existence de relations latentes non activées. L'accent sera mis sur 3 points essentiels : sa relation avec son directeur de thèse, ses habitudes et lieux de travail impliquant ou non l'activation de relations avec d'autres chercheurs « proches » en formation ou confirmés, ses contacts avec des chercheurs n'appartenant pas directement à son entourage scientifique immédiat.

De 2006 à 2011, nous avons conduit une recherche-action auprès de doctorants de sciences humaines de l'Université Toulouse le Mirail volontaires pour une présentation des principaux travaux disponibles sur la question des relations et des ressources et pour se prêter à un certain nombre d'exercices destinés à la fois à leur faire prendre conscience de leur univers professionnel, et à imaginer les modalités de sa mobilisation. L'effectif total fut de 41 étudiants se répartissant comme suit : 7 en 2008-2009, 8 en 2009-2010, 9 en 2010-2011, 14 en 2011-2012 et 3 cas réalisés avec des doctorants de notre laboratoire, que nous avons soumis aux mêmes questions. Parmi ces 41 étudiants, 35 furent présents à toutes les séances et nous ont permis de suivre systématiquement l'évolution de leurs « réseaux en train de se faire». 
Tableau 1 : principales caractéristiques des étudiants suivis

\begin{tabular}{|c|c|c|c|c|c|}
\hline nationalité & effectifs & spécialité & effectifs & année thèse & effectifs \\
\hline française & 18 & sociologie & 13 & 1ère & 17 \\
\hline vietnam & 4 & histoire & 6 & 3ème & 9 \\
\hline cameroun & 2 & économie & 3 & 2ème & 7 \\
\hline gabon & 2 & architecture & 3 & 4ème & 1 \\
\hline tunisie & 1 & sciences de l'éducation & 2 & 5ème & 1 \\
\hline algérienne & 1 & dynamiques rurales & 2 & & \\
\hline cambodge & 1 & littérature & 1 & & \\
\hline honduras & 1 & psychologie & 1 & âge médian & 28,5 \\
\hline niger & 1 & tourisme & 1 & écart moyen & 4,32 \\
\hline sénégal & 1 & anthropologie & 1 & & \\
\hline haiti & 1 & archéologie & 1 & & \\
\hline chine & 1 & géographie & 1 & & \\
\hline non renseigné & 1 & & & & \\
\hline
\end{tabular}

La formation proposée à ces doctorants partait de deux constats complémentaires : d'une part, dans notre équipe de recherche en sociologie, le récit par deux doctorants des modalités utilisées pour rechercher un financement de thèse et d'autre part, l'hypothèse d'une sous-utilisation des ressources relationnelles des doctorants de l'Université, eu égard aux pratiques des diplômés des Écoles d'ingénieur ou de Commerce. En effet, s'agissant des deux doctorants, qui ont cherché un ou plusieurs partenaires financiers pendant une année, il est avéré que plusieurs éléments positifs avaient joué en leur faveur: pour la première étudiante, le fait de disposer d'un diplôme d'ingénieur a constitué une marque de confiance auquel l'un des partenaires, lui-même ingénieur, a été sensible. La qualité du dossier et l'engagement d'un premier partenaire ont fait le reste. Remarquons toutefois que le contrat de financement nécessitait une triple localisation, rendue possible par la présence de son cercle familial élargi dans l'un des trois lieux. Pour le second, les relations activées provenaient du réseau universitaire de sa Directrice de thèse et de la mobilisation impromptue de relations plutôt faibles et indirectes, alors que les premières démarches plus directes, liées au sujet de recherche, étaient vaines, notamment celles qui tentaient d'enrôler des partenaires étrangers au 
monde de la recherche en sociologie. De ces deux histoires de recherche de ressources financières et relationnelles, nous avions tiré quelques hypothèses sur l'activation des réseaux par les doctorants : leurs parcours antérieurs peuvent constituer une force ou une faiblesse pour intéresser des organismes prêts à financer une thèse, les relations des encadrants de la thèse sont importantes et enfin, la mobilisation des chaînes de recommandation, rendues encore plus opérationnelles grâce à l'internet, n'est ni aisée ni automatique. Elle peut nécessiter la compréhension des codes relationnels en vigueur dans l'univers visé.

Sur l'hypothèse d'une sous-utilisation des ressources relationnelles des doctorants de l'Université, confirmée par le directeur de l'école doctorale, l'idée était d'amorcer avec les participants à l'atelier, une recherche sur le devenir des anciens docteurs de l'Université du Mirail. En effet, le manque d'information, dans les laboratoires, sur les parcours professionnels de docteurs en Sociologie ou dans des disciplines proches (ethnologie, anthropologie, etc.) autres que les carrières académiques classiques nous paraissait un premier obstacle à la mobilisation de ressources relationnelles, telle que peuvent la déclencher les diplômés d'Écoles d'ingénieur et de Commerce (Bès \& Chaulet, 2012), surtout dans le cas d'une recherche de financements ciblés, autres que les allocations de recherche, obtenues auprès des laboratoires scientifiques. Nous partions de l'idée que les doctorants pourraient tirer parti de l'identification de diplômés leur ressemblant, c'est-à-dire d'anciens doctorants, ayant poursuivi une thèse à l'Université de Toulouse Le Mirail, sur un sujet et une disciplines proches et ayant réalisé leur carrière, ailleurs qu'à l'Université. Concrètement, les différentes bases de données bibliographiques et les réseaux sociaux numériques permettent assez facilement de retrouver les traces de doctorants ainsi que leurs Curriculum 
Vitae, comme l'a montré en séance Nathalie, à partir d'un cas réel d'un ancien doctorant, devenu salarié d'un grand groupe, et dont il est assez facile de reconstruire la carrière depuis qu'il est docteur de l'Université.

On suggérait alors aux doctorants de contacter ces « anciens » et de reconstituer leur parcours depuis le doctorat au cours d'entretiens. Cette prise de contact aurait aussi comme finalité, une mise en confiance de l'étudiant par identification de parcours de réussite et une familiarisation avec les codes langagiers et sociaux à l'œuvre dans ce milieu, comme le font, par exemple, les élèves d'écoles d'ingénieurs et de commerce au cours de leurs études, au moment de conférences et autres rencontres avec les anciens diplômés (Lazuech, 2000). Il était possible aussi que ces anciens proposent des pistes de financement.

Avec le double objectif d' « aider le doctorant à connaître son réseau et repérer des docteurs qui lui ressemblent », nous avons ouvert une formation doctorale en septembre 2008, intitulée «Club de chercheurs débutants en sciences humaines », dont les objectifs étaient présentés de la manière suivante :

"1. créer une dynamique en rassemblant régulièrement des doctorants à la recherche de partenaires financiers pour une thèse avec des professionnels du marché du travail et de l'emploi immergés dans le milieu de la recherche pour qu'ils ne soient plus isolés, qu'ils mutualisent les informations existantes, les compétences, les contacts de chacun.

2. Contacter les anciens élèves de l'Université au plus près des spécialités des participants de manière à savoir ce qu'ils sont devenus, où ils exercent, quels sont leurs parcours. 
3. Ce projet se veut une recherche-action se double d'un objectif d'étude de la manière dont se sont intégrés les anciens doctorants. Une publication pourra être envisagée sur la mobilisation des réseaux personnels dans la recherche de financement de thèse ${ }^{4}$.

La formation ${ }^{5}$ devait concerner en priorité les doctorants en première année, et nous avions pensé que leur demande principale serait la recherche de financement/ et ou d'organismes susceptibles de fournir un emploi. Ainsi, la première version de l'atelier était très axée sur la recherche d'un financement de thèse pour les doctorants en début de thèse et sur l'obtention d'un emploi pour ceux, qui se trouvaient en situation de finir. Il est important de souligner que la formation n'était pas obligatoire, qu'elle faisait partie du programme des formations doctorales ${ }^{6}$ et qu'elle entrait donc en concurrence avec d'autres thèmes aussi variés que : l'espace des sciences sociales, le fait religieux, les espaces ruraux, la maîtrise des tableaux, la formation à des logiciels, etc.

Nous avons rapidement constaté que les inscrits ne correspondaient pas nécessairement à ces deux profils, étant déjà soit très avancés dans leur thèse avec souvent des financements, soit en dernière année, sans besoins précis. Par ailleurs, il s'est très vite avéré impossible de leur demander de mener des entretiens avec les anciens de l'Université, bon nombre d'entre eux ne maîtrisant pas les techniques d'entretien sociologique, et n'étant pas disponibles pour les acquérir. Dès la deuxième année, il s'est

4 Extrait du document envoyé à l'Ecole Doctorale TESC pour présentation de la formation.

5 Il est important de souligner que la formation n'est pas obligatoire, qu'elle fait partie du programme des formations doctorales et qu'elle entre donc en concurrence avec d'autres thèmes aussi variés que : l'espace des sciences sociales, le fait religieux, les espaces ruraux, la maîtrise des tableaux, la formation à des logiciels, etc.

6 les étudiants, inscrits en thèse, étant tenus d'accomplir un certain volume horaire, certains d'entre eux ont participé à notre formation, pour valider des heures. 
trouvé que les étudiants inscrits dans le «club» étaient dans la situation intermédiaire, qui n'était ni le début de la thèse ni sa fin. Mais les participants exprimaient tous une satisfaction vis-à-vis de la formation, estimant $\mathrm{y}$ avoir appris beaucoup sur le fonctionnement du marché du travail et particulièrement du milieu de la recherche. Le deuxième objectif a donc été abandonné tel quel au profit d'une recherche d'autres types de ressources nécessaires à la conduite de la thèse. En septembre 2009, la formation a trouvé son intitulé définitif sous l'appellation « Réseaux et insertion professionnelle des doctorants ». Il était alors clairement mentionné que nous allions utiliser les méthodes des réseaux sociaux : «La participation des étudiants consistera ensuite à analyser, avec l'aide des intervenantes, leurs propres appuis relationnels au sein de leur équipe de recherche et au sein de la Communauté scientifique afin de mettre en évidence les ponts relationnels nécessaires ».

Les doctorants cherchaient rarement un financement ou un emploi mais plutôt l'accès à des ressources difficilement accessibles et variées, allant d'un tableau peint par un artiste du 18ème, jusqu'à un organisme international en passant par un chercheur réputé. C'est exactement le cas de Bastien, qui a influencé grandement la bifurcation de l'objectif de la dernière séance de l'atelier : celui-ci nous fait part de sa volonté de rentrer en contact avec une personne-cible, journaliste à la retraite mais témoin d'événements politiques et tragiques particuliers et qui pourrait détenir des archives, documents et récits inédits. Ainsi, dès la deuxième année, nous laissions le doctorant formuler le type de ressources auxquelles il voudrait accéder en acceptant qu'elle puissent évoluer au cours des séances ou qu'elles puissent être multiples.

Nous avons donc adapté notre proposition et orienté la partie recherche de cette action vers le fait de comprendre la manière dont ils percevaient leur réseau professionnel, in 
abstracto et de facto et celui des «appuis » universitaires les plus proches (surtout directeur de thèse ou co-directeur). Nous ne détaillerons pas ici les conseils donnés au fur et à mesure en matière de recherche d'emploi ou les explications sur le fonctionnement du milieu de la recherche. Il s'est agi de comprendre d'abord l'entourage du doctorant et de l'aider à appréhender cet environnement professionnel. Dans un premier temps, les questions posées seront donc: Quelles sont ses relations avec son directeur de thèse ? avec son laboratoire d'accueil ? avec d'autres chercheurs ? avec d'autres étudiants ? Dans un deuxième temps, nous aidions le doctorant à appréhender cet entourage professionnel en prenant conscience de son réseau, en lui apprenant à tisser un lien professionnel. Est-il isolé et si c'est le cas, pourquoi ? Nous utilisons les matériaux recueillis à cette occasion, composés de réseaux mais aussi des témoignages recueillis au cours des séances de formation. Celles-ci, à partir de la deuxième année, se déroulaient en quatre temps espacés de plusieurs semaines : une journée complète d'apports théoriques et pratiques sur le marché du travail, les réseaux et le milieu de la recherche, puis trois demi-journées sur des exercices pratiques.

A l'issue de la formation distillée à ces quatre promotions, nous disposons des éléments suivants :

Des traces écrites obtenues en co-construction avec les participants au cours des séances qui sont des tableaux de générateurs de noms, des réseaux personnels et des chaînes de personnes dessinées, appelés aussi graphes de réseaux, des éléments permettant de construire les réseaux des directeurs de thèse (CV par exemple, liste des publications) et enfin, une liste de ressources propres à chaque doctorant, associées, selon lui, à la meilleure manière de parvenir à les obtenir. Ainsi, a-t-on collecté en séance des éléments sur le doctorant avec son CV (âge, spécialité, année d'inscription en thèse, 
nationalité, parcours de formation et parcours professionnel) et quelques éléments déclaratifs sur les différents endroits de conduite de la thèse (lieu de travail, d'écriture, fréquentation du laboratoire, clé d'un bureau, etc.) et sur le mode de financement de la thèse (allocation, emploi alimentaire, soutien familial). Nous avons donc collecté ces éléments au cours d'une observation participante par notation et observation des réactions, des réticences, de commentaires en situation, des obstacles rencontrés et des dispositions corporelles et d'interaction sous forme de prises de notes en direct et à chaud par l'une d'entre nous. Nous avons aussi conduit quelques entretiens individuels complémentaires dans les locaux de notre laboratoire, en face à face, toujours sur le thème de leurs ressources sociales.

\section{Les réseaux d'étude des doctorants}

Les données collectées auprès de chaque doctorant sur son réseau personnel se composent de deux types de traces : un listing de personnes caractérisées par des variables précisées dans le tableau ci-après et un graphe des relations entre les personnes indiquant aussi la nature des cercles sociaux (Universités, entreprises, voisinage, etc.) dans lesquelles ces personnes ont été rencontrées. Ces deux matériaux ont été complétés par les commentaires et remarques des participants, recueillies sur le vif.

Une grille a été élaborée en vue d'obtenir une liste de personnes avec lesquelles les doctorants parlent de leurs thèses. Ce critère sert donc de générateur de noms. Puis, les étudiants devaient donner, pour chaque nom cité, des éléments sur la sphère sociale à laquelle se rattache la personne et son statut professionnel, des éléments qualifiant le 
lien (intensité, fréquence, nature, contenu des conversations, ancienneté, contexte de rencontre) ainsi que l'existence ou non de relations d'interconnaissance entre les personnes citées. Nous nous étions inspirées d'une grille similaire utilisée par des chercheurs québecois travaillant avec des joueurs sur leur addiction en leur faisant prendre conscience des personnes susceptibles de les influencer dans leur réseau (Biron et al. 2008).

Tableau : Liste des personnes avec lesquelles « le doctorant parle de sa thèse

\begin{tabular}{|c|c|c|c|c|c|c|c|c|c|}
\hline Personne & $\begin{array}{l}\text { Sphères sociales } \\
\text { (familie, } \\
\text { université, labo...) }\end{array}$ & $\begin{array}{l}\text { Nature des relations } \\
\text { (cousin, voisine, } \\
\text { directeur de thesse, } \\
\text { collegue, ami) }\end{array}$ & $\begin{array}{l}\text { Statut professionnel, } \\
\text { employeur, lieu de } \\
\text { travail (employé, } \\
\text { EDF, Tarbes) }\end{array}$ & 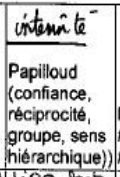 & $\begin{array}{l}\text { Fréquence (1 } \\
\text { fois/semaine, } \\
\text { fois/mois, ...) }\end{array}$ & $\begin{array}{l}\text { Contenu des } \\
\text { conversations } \\
\text { (échanges } \\
\text { d'informations, } \\
1 \text { soutien, } \\
\text { encadrement,...) }\end{array}$ & $\begin{array}{l}\text { Ancienneté } \\
\text { de la relation }\end{array}$ & $\begin{array}{l}\text { Contexte de } \\
\text { création de la } \\
\text { relation (dans } \\
\text { quel cadre avez } \\
\text { vous roncontré la } \\
\text { personne la } \\
\text { premiere fois ?) }\end{array}$ & $\begin{array}{l}\text { Qui est en } \\
\text { relation avec } \\
\text { qui ? (indiquez le } \\
\text { numéro des } \\
\text { personnes } \\
\text { citées) }\end{array}$ \\
\hline 1 Ronan & famille & Fiancé & $\begin{array}{c}\text { Charge de lom } \\
\text { U IMM }\end{array}$ & $\begin{array}{l}\text { Len joct } \\
\text { con fibme }\end{array}$ & 7 gais/sem & $\begin{array}{l}\text { Echanges } \\
\text { sourient }\end{array}$ & gans & Loing & $1 / 2$ \\
\hline 2 JEF & Famille & Parent & Retrátel & $\begin{array}{l}\text { lien tout } \\
\text { conftan } 6\end{array}$ & $|f| s 0 m$ & sourient & 30 ans & - & $2 / 1$ \\
\hline${ }_{3}$ Lavence & Labo/ UTTI & Co-Directeur & $\Pi D C$ & lien moyent & $2 \mathrm{f} / \mathrm{mois}$ & encade ${ }^{T}$ & 4 ans & UTTI & 4 \\
\hline 4 JPfere & Labo/UTा & Directeus & Prog. & $\begin{array}{l}\text { Wen factore } \\
\text { hiesaichio }\end{array}$ & 3 flans & $\begin{array}{l}\text { encaduat } \\
\text { Rierarchis }\end{array}$ & 4 ans & जाT & 3 \\
\hline 5 Daniela & 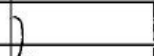 & collegues| & Chougé d'eturde & & & - & & 7 & $3^{3 / 4 / 6 / 718)}$ \\
\hline 6 harine & laboum & ani & & & $1.6 \mathrm{sem}$ & Southent & & $U T M$ & 21411 \\
\hline 7 Diana & & & Doctoraut & & & & & & $67 / 8$ \\
\hline $\begin{array}{l}\text { Esteile } \\
8 \text { Tristan }\end{array}$ & & & D & & $\int$ & & & & $1+4+10$ \\
\hline 9 Natacha 7 & & & Chargés Etude? & lien & 7 & & 7 & & $10 / 3 / 4 / 1115$ \\
\hline 10 Nicolas & $\left.\begin{array}{l}\text { Labo } \\
\text { exteriver }\end{array}\right\}$ & Colleques & $\begin{array}{l}\cap D C \\
\text { cherchere }\end{array}$ & foinde & & & 3aus & 077 & $9 / 3 / 4 / 11 / 5$ \\
\hline 11 Alimi & $J$ & & Vaué MDC & & J & $J$ & & & $10 / 9 / 4 / 3 / 5$ \\
\hline 12 Amélie & fouille & amie & cade AGría & Cou fout & $3 \mathrm{f} /$ mois & échouiges & souss & école & 112 \\
\hline
\end{tabular}

Les doctorants ont donc, à notre demande, opéré arbitrairement une partition dans leur réseau personnel, afin de lister les personnes « avec lesquelles ils parlent de leur thèse ». On obtient ce que l'on pourrait appeler le réseau personnel d'étude ou le réseau personnel doctoral selon que l'on considère le doctorant plutôt comme un étudiant ou 
bien comme un travailleur. Justement, la réalité se situe entre ces deux situations étude/travail et la description de leur réseau personnel renvoie à cet entre-deux relationnels.

Dans l'exercice d'énonciation des noms « des personnes avec lesquelles on parle de sa thèse », les participants ${ }^{7}$ ont cité en moyenne 12 personnes avec un écart-type de $4^{8}$. On peut donc souligner la taille réduite de ce réseau, soit parce qu'il est dédié spécifiquement à la thèse et donc qu'il n'est qu'une partie du réseau personnel de l'étudiant ${ }^{9}$, soit parce que l'étudiant oublie de mentionner des personnes. Quoiqu'il en soit, ce « petit » réseau personnel peut laisser présager un accès restreint aux ressources informationnelles (bibliographie, terrain, données, méthodes, échanges, contacts directs avec des chercheurs, controverses, etc.) dont ont besoin ces étudiants.

Parmi les personnes citées, on trouve systématiquement ${ }^{10}$ le directeur (la directrice) de thèse ainsi que le co-encadrant (e). Souvent, un autre professeur, membre de l'équipe du directeur de thèse est également noté dans les personnes « ressources » de la thèse. Mais hormis ces contacts - directeur de thèse + éventuellement un autre professeur -, on trouve très peu d'universitaires, ce qui souligne, la forte dépendance du doctorant vis-àvis de son encadrant et la taille réduite de son réseau «d'étude». Il se peut que ce résultat soit lié aux disciplines scientifiques des doctorants concernés, centrées sur les

$7 \quad$ Il s'agit des 29 étudiants ayant participé aux 3 dernières formations car nous ne disposons pas du nombre pour la première promotion.

$8 \quad$ Ces moyennes ont été obtenues à l'issue de 2 séances successives : la moitié environ des stagiaires ont rajouté 2 ou 3 personnes, en général des doctorants de leur laboratoire entre les deux séances.

9

Dans l'ouvrage sur la vie en réseau (Bidart, Degenne \& Grossetti), le nombre de relations citées par les personnes âgées de 18 à 25 ans est de 26 et de 28 pour celles âgées de 26 à 45 ans. Plus précisément, les titulaires d'un diplôme Bac +4 citent 36.6 personnes.

10 Dans un seul cas, il n'est pas mentionné par une étudiante, qui dit l'avoir oublié par « étourderie ». 
sciences humaines et moins collectives que d'autres sciences.

Dans l'ensemble des personnes universitaires citées, la part locale est très forte : elle comporte, soit une seule ville (Toulouse), soit deux (Toulouse et la ville où l'étudiant a fait ses études en premier et deuxième cycle universitaire). Nous avons fait part aux étudiants, de la quasi-absence de contacts dans la capitale française alors que le nombre de chercheurs en Sciences en Sociales à Paris et donc de séminaires, colloques, etc. est très élevé.

Dans le tableau des citations, la présence d'acteurs liés au terrain d'étude du doctorant est variable et dépend du type de ressources initiales dont disposait le doctorant avant de commencer sa thèse (employé dans ce secteur d'activité par exemple) et également du degré d'avancement de la thèse.

Par ailleurs, les membres de la famille sont mentionnés comme soutien financier à la conduite de la thèse. Les étudiants en thèse citent aussi un nombre important de leur pairs, à savoir d'autres doctorants, auxquels ils attribuent un rôle de soutien moral et avec lesquels ils partagent à la fois des ressources cognitives "pures» (livres, méthodes, idées, etc.) mais également une expérience commune.

Du graphe du « réseau dessiné » par le doctorant, il ressort quelques éléments saillants :

- d'une part, le directeur de réseau, s'il est positionné dans la sphère de l'Université, n'est pas nécessairement placé à côté du doctorant et même parfois beaucoup plus loin, parce qu'il est une ressource isolée que le dessinateur relie 
difficilement avec les autres membres de son réseau : soit, il ne sait pas quelles sont les personnes connues par le directeur de thèse et donc les liens autour de ce dernier ne sont pas renseignés, soit au contraire, systématiquement, sans réfléchir, il dessine une clique qui relie le directeur de thèse à tous les autres membres permanents du laboratoire.

- d'autre part, le réseau est très cliqué, c'est-à-dire qu'il comporte des groupes relativement indépendants de personnes fortement reliées entre elles. On peut remarquer deux types de «trous structuraux »: ceux qui apparaissent entre l'Université d'origine - si l'étudiant a suivi le début de son cursus ailleurs -, et l'Université de Toulouse et ceux existant entre l'équipe de recherche et le terrain d'étude. Dans les deux cas, c'est l'étudiant qui fait le lien entre ces cercles relativement étanches.

- le « réseau dessiné » comporte 4 composantes dans le cas le plus fréquent et jusqu'à 6 : la sphère familiale apparaît en bonne place mais englobe peu de personnes ; les amis dont la moitié sont à l'Université représentent le cas rare de recouvrement de deux sphères (amitié, Université) ; l'Université est la troisième sphère d'activité évoquée dans laquelle une distinction s'opère entre les relations entre pairs, qui sont celles des doctorants entre eux et les relations de l'étudiant avec les professeurs et les chercheurs, vécues comme fondamentalement hiérarchiques. La quatrième sphère d'activité évoquée est celle des relations professionnelles hors thèse, qui renvoient à un emploi antérieur ou concomitant à la thèse, mais souvent vécu comme un « job alimentaire ». Les deux dernières sphères que l'on peut trouver et qui présentent une taille et densité moins fortes 
que les quatre premières sont le premier lieu de formation de l'étudiant (s'il a suivi ses premières années de formation ailleurs qu'à l'Université de Toulouse) et la sphère d'activité qui englobe les archives et les relations nouées sur le « terrain ». Cette dernière comporte peu d'individus bien qu'elle constitue une ressource informationnelle essentielle pour le doctorant.

En examinant les relations manquantes ou faiblement présentes dans les réseaux personnels des doctorants, on observe une distinction entre 3 types de réseaux ${ }^{11}$ :

- Ceux des «passagers clandestins » des laboratoires, qui travaillent seuls en dehors du laboratoire, ont un réseau d'aide à la thèse très restreint. Leur directeur de thèse est une relation faible et peu fréquente. De même, ils ne mentionnent quasiment pas de « collègues » universitaires (doctorants ou chercheurs permanents). Souvent, ils n'ont pas réalisé leur formation antérieure à l'Université de Toulouse. On trouve le cas du doctorant étranger isolé ou celui du doctorant dont les ressources de travail ne sont pas à l'Université car très spécifiques (archives, surtout) et qui commence sa thèse. Leurs réseaux peuvent être amicalement «riches» mais peu fournis en chercheurs ou enseignants. Les plus mal dotés d'entre eux n'ont pas assez de liens avec leurs terrains et manquent aussi de ressources de ce côté-là. A l'inverse, on trouve aussi dans ces cas de petits réseaux, les doctorants, déjà professionnels, qui font une thèse en parallèle ou lors d'une période «suspendue » de leur activité professionnelle. Certains ne participent pas nécessairement à la vie du laboratoire. Dans les deux cas, le réseau est très cliqué et les personnes des différentes sphères

11 Nous avons déjà utilisé une typologie analogue lors des Journées Nationales des Observatoires de l'Enseignement Supérieur, Cf. Marie-Pierre Bès \& Nathalie Chauvac, « les réseaux des doctorants. Le cas de l'Université de Toulouse le Mirail », Université Toulouse Capitole, 23 Juin 2011, http://oatao.univ-toulouse.fr/4977/. 
n'ont pas de relations entre elles. Nous qualifierons cette rubrique de «petit réseau cliqué».

Schéma 1 : un petit réseau d'étude cliqué, peu lié à l'Université

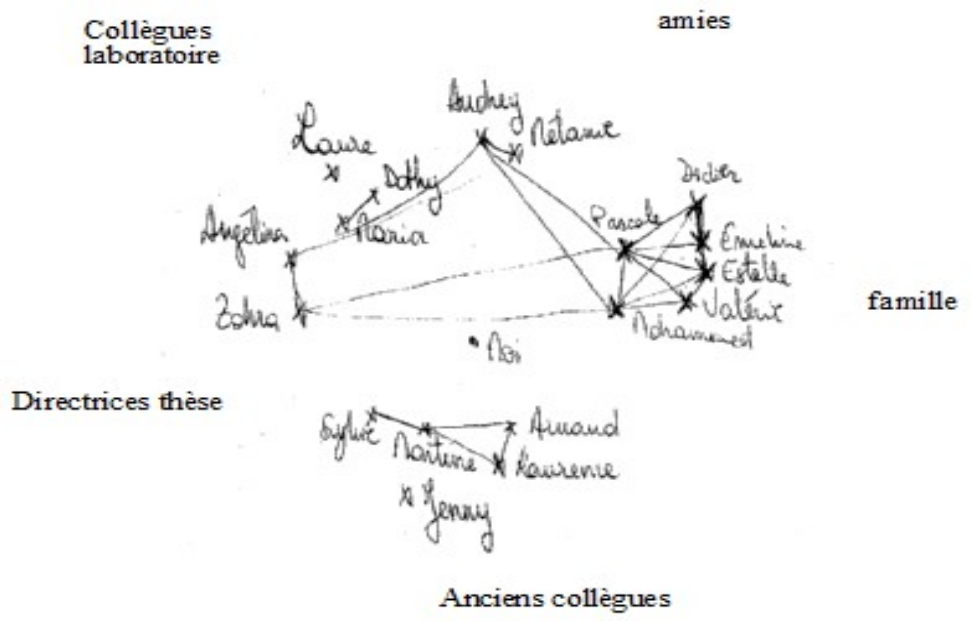

- Les réseaux des doctorants bien insérés dans le milieu de la recherche et/ou de la structure qui les finance (allocation de recherche, bourse étatique émanant de différents organismes, financement CIFRE, etc.). Ceux-là citent de nombreuses personnes qui peuvent les aider pour leur thèse et notamment des interlocuteurs collègues sur lesquels ils vont s'appuyer à la fois, pour avoir des informations essentielles pour la suite de leur parcours et pour être soutenus ou guidés. Ce réseau est qualifié d'universitaire. 
Schéma 2 : Réseau universitaire

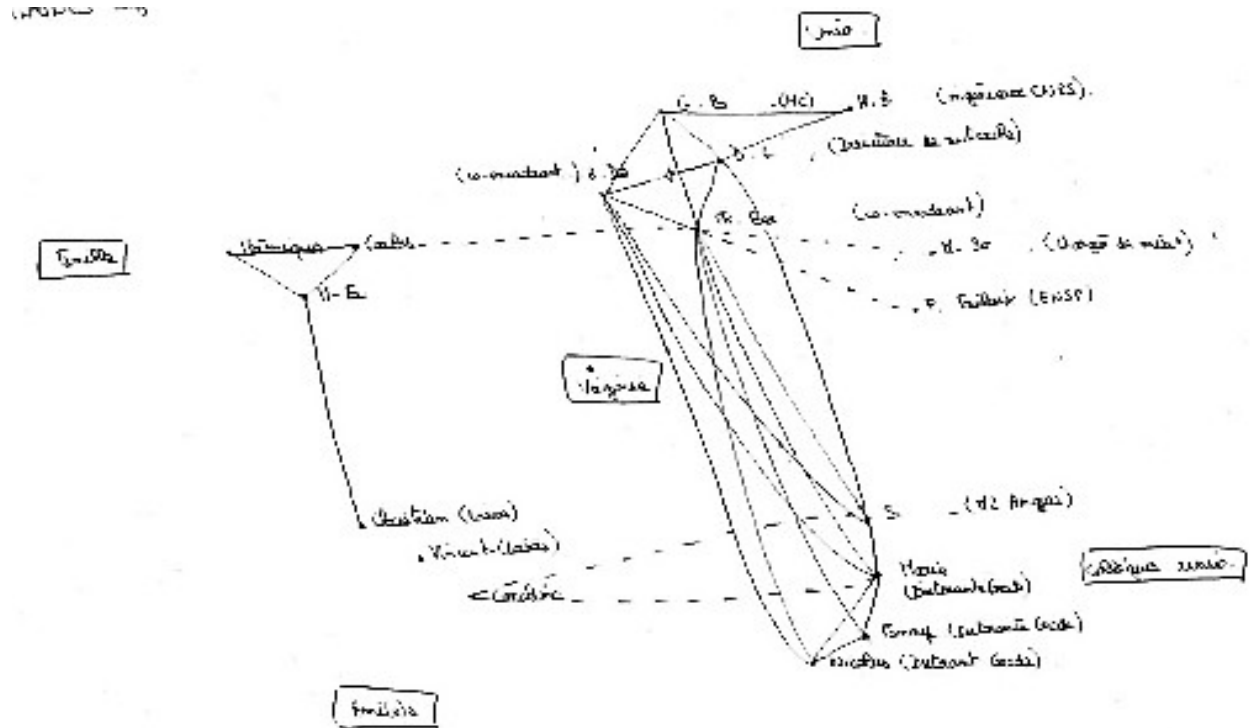

- Le troisième type de réseau est celui des doctorants qui sont à l'interface entre le monde universitaire et le monde social auquel ils s'intéressent (peintres, immigrés, consommateurs, écrivains, architectes, etc.). Ce sont ceux qui ont des relations avec les deux ressources essentielles pour une thèse : des relations universitaires et des relations avec les personnes appartenant à leur «terrain». Ils sont en position de centralité d'intermédiarité. Dans ces réseaux, les nœuds et les relations sont plus nombreux mais chaque cercle social (famille, amis, Université, monde professionnel) est représenté à parts égales, sans qu'il soit possible de dégager une quelconque prépondérance en termes de personnes citées. 
Schéma 3 : Réseau dense et équilibré recherche/terrain

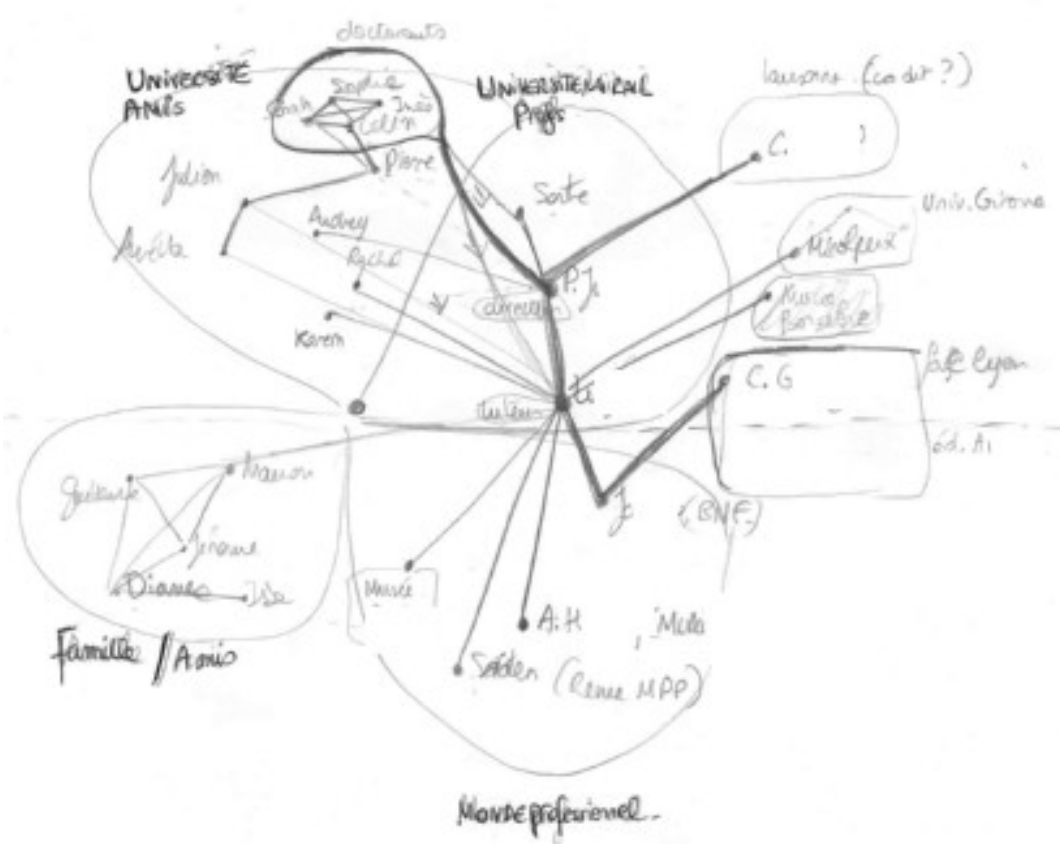

Au cours de la formation et de l'observation des graphes et tableaux de générateurs de noms, nous n'avons porté aucun jugement de valeur sur la performance de ces types de réseaux vis-à-vis de la conduite de la thèse, notamment parce que les discussions n'ont jamais porté sur les avancées de la thèse, proprement dites (données collectées, méthode d'analyse, outils utilisés, bibliographie, éléments théoriques mobilisés, articles écrits, présentations effectuées, etc.) et parce que, d'autre part, nous pensons que les disciplines (archéologie, sociologie, histoire, psychologie, etc.) ont des traditions de recherche, qui pèsent sur les manières de travailler des doctorants avec les autres.

$\mathrm{Au}$ sein de notre effectif, la moitié des doctorants correspondent au type «petits 
réseaux ", ce qui confirme que certains étudiants se soient inscrits à notre formation, dans un objectif assez utilitariste. Environ 1/5 correspondent à la catégorie du réseau, plutôt orienté vers le monde de la recherche tandis que 1/5 ont un réseau dense entre la recherche et le terrain ${ }^{12}$.

Nous mettrons à l'épreuve cette typologie en trois réseaux (petit réseau cliqué, réseau universitaire et réseau dense) dans la dernière partie sur les cibles-ressources.

\section{Le réseau du directeur de thèse tel qu'il est élaboré par le doctorant}

Comme évoqué dans la première partie, l'étape suivante pour l'étudiant fut de chercher des éléments permettant de «tracer » le réseau relationnel de son directeur de thèse, en se basant essentiellement sur un Curriculum Vitae et des données publiques. Il s'agit d'obtenir une liste de ses principaux collaborateurs c'est-à-dire ses co-auteurs et des personnes avec lesquelles il a partagé des activités d'enseignement ou de coordination pédagogique. De cet exercice et de nos observations lors de la conduite de cette étape, il en ressort les difficultés suivantes :

- en premier lieu, soulignons la difficulté, pour ces doctorants, de construire ce réseau : la liste des collaborateurs officiels du directeur de thèse est longue à établir et ne donne pas une information classée et hiérarchisée, les interconnaissances entre chercheurs sont en partie informelles, au sens qu'elles ne se traduisent pas nécessairement par des collaborations explicites. Par exemple, les doctorants témoignent avoir déjà entendu leurs directeurs de thèse lister des

12 Pour quelques-uns, nous ne disposions de trop peu d'éléments pour classer le réseau ou le tableau de générateurs de noms dans l'une des catégories. 
connaissances dans le milieu académique sans qu'il soit possible d'en trouver la trace « officielle ». Ainsi, un étudiant parle de « réseau caché » et enchaîne, sur ce point, avec le cas de son Directeur de thèse qui a fait ses études à New York et qui a gardé des liens avec d'anciens étudiants dont il lui a transmis les coordonnées : «je ne les aurais jamais trouvés, s'il ne m'avait pas donné leurs coordonnées ». D'autre part, des participants à la formation ont souligné leur étonnement face à la taille réduite du réseau de leur directeur. Un doctorant s'exclame : «je m'attendais à quelque chose d'énorme, vu que quand on va au restaurant avec lui, il dit bonjour à tout le monde, il connaît tout le monde (...) Or je me suis rendu compte de la petitesse de son réseau (..), moi aussi je m'étonne de n'être que son deuxième doctorant $»$. Évidemment l'écart est réel entre les personnes connues d'un enseignant-chercheur confirmé, surtout sur son lieu de travail et à travers ses différentes activités (enseignement, coordination pédagogique, administration de projets, direction de thèses, responsabilités institutionnelles, etc.) et les personnes que l'on peut appeler des collaborateurs explicites et publics - co-auteurs, par exemple - mais, cependant, il y a quand même un fort décalage entre le réseau réel obtenu et le réseau « imaginé » par le doctorant, comme si cet exercice s'accompagnait d'une sorte de « désenchantement du directeur de thèse » ou d'un processus de démystification de la fonction du Directeur de thèse, plutôt associée à une position sociale haute dans l'Université. Ainsi, Melissa découvre qu'elle est la première doctorante de sa Directrice de thèse et explique cela dans une attitude à la fois détendue (elle rit) mais également un peu crispée en expliquant : «je me suis donc rendue compte qu'elle était un peu novice dans la direction de thèses, ça me fait un peu peur». 
- D'ailleurs les doctorants, qui ont fait l'effort de compulser des données relationnelles sur leurs directeurs de thèse, ne les ont pas informés de cette initiative et paraissent aussi gênés par cette démarche : ils ont soit, honte («de faire la fouineuse» comme le dira une doctorante»), soit, l'impression de remettre en cause la légitimité de leur Directeur. Evidemment, on peut penser qu'il s'agit aussi pour eux, de se rendre éventuellement compte de leur méconnaissance préalable du milieu et de leur mauvais choix. Le doctorant est en situation de subordination avec son directeur de thèse et avec d'autres permanents du laboratoire et de l'institution. Il n'ose pas lui avouer qu'il cherche des informations sur lui, qui pourraient remettre en cause sa position symbolique dominante et du coup, pourrait aussi lui révéler un niveau d'insertion dans la communauté universitaire plus faible que celui auquel il s'attendait.

Schéma 4 : Le réseau professionnel d'un Directeur de thèse

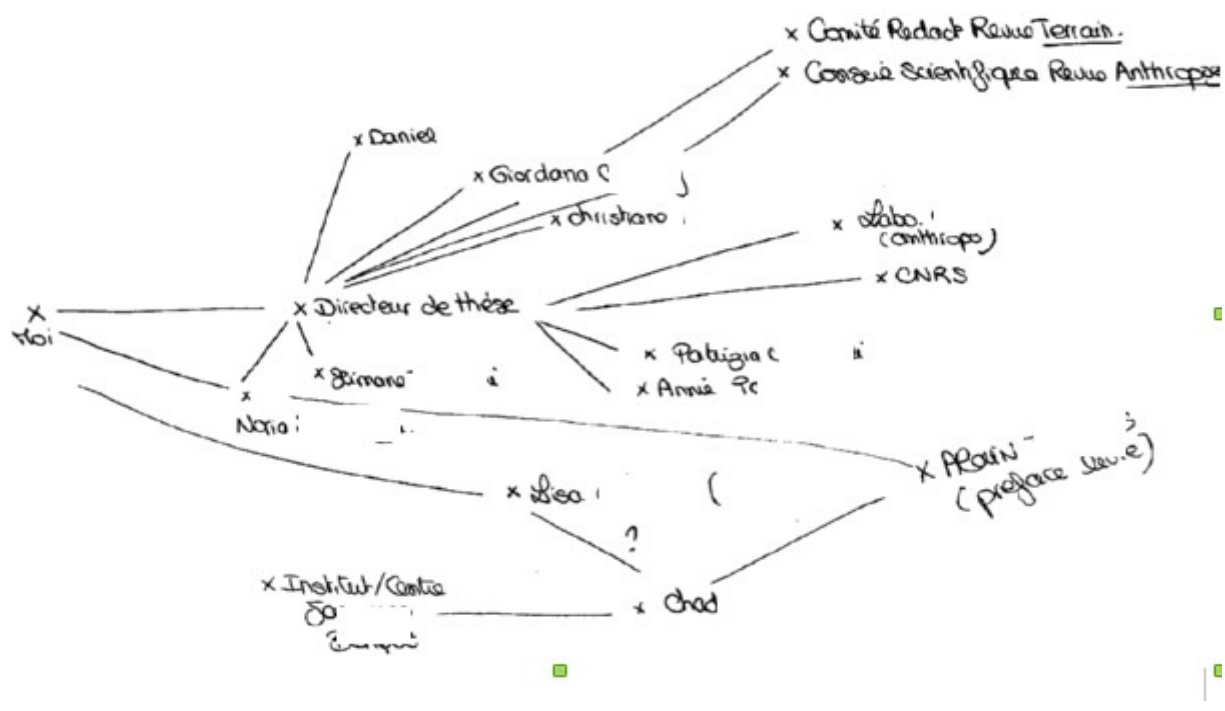

En listant les collaborateurs de leur encadrants et également les niveaux institutionnels 
auxquels ils sont rattachés (Université, Comité de rédaction d'une revue, organisme de formation, etc.), les doctorants découvrent et expriment le fait que « c'est vraiment un petit monde, c'est-à-dire que tout le monde se connaît ». Ils découvrent, au cours de cet exercice, la concentration des rôles et des pouvoirs et donc les probables échanges d'information entre ces personnes. Certains en profitent pour démarrer une sorte de cartographie de leurs spécialités scientifiques, dans laquelle ils peuvent placer leurs encadrants, leur équipe mais aussi bien d'autres chercheurs en France ou ailleurs.

La conscience que le doctorant peut avoir de relations inexistantes ou « négatives », que son Directeur de thèse a avec d'autres chercheurs de sa spécialité, est rare. Pour la grande majorité des doctorants que nous avons formés, ils connaissent très peu le réseau de leur directeur de thèse, donc a fortiori, ses éventuelles relations conflictuelles ou inexistantes.

Dans un seul cas particulier, la doctorante, prénommée Hélène, savait que son directeur de thèse n'était pas favorable à l'établissement d'une relation avec une chercheuse. Le contexte est celui du montage d'un projet de recherche, confié à l'étudiante, qui a déjà une expérience professionnelle dans le secteur d'application de la recherche en sociologie et qui a repris ses études aux alentours des 35 ans. L'étudiante se retrouve devant une difficulté qu'elle qualifie elle-même de « relationnelle» : une chercheuse étrangère (liée à un Institut Français de son pays ) voudrait rentrer dans le réseau de collaboration de son Directeur de Thèse et participer à ce projet. Or, la doctorante comprend vite que son Directeur de thèse ne veut pas «pousser le lien plus loin avec elle parce qu'il ne la trouve pas sérieuse », et qu'il ne partage pas son approche du sujet par l'entrée de la santé. Elle sait, par ailleurs, que son Directeur de thèse a déjà 
rencontré cette chercheuse et qu'il l'a aidée à « faire passer un article » et qu'il est donc plutôt dans une position de domination. L'embarras d'Hélène provient de sa volonté de ne pas se couper des contacts avec ce pays, qui est le sujet de sa thèse et en particulier, d'éventuels liens avec des chercheurs. Elle pressent que ces derniers vont chercher à savoir si leur équipe est en lien avec la chercheuse en question et que cette première collaboration pourrait alors être un signal positif pour la suite des contacts avec leur équipe. Elle répète, gênée : «y a un blocage, ça passe pas et je sais pas quoi faire ».

Pendant la formation, au moment où la discussion collective a porté sur ses aspects et où Hélène a exposé publiquement son cas, une autre doctorante a pris alors la parole et demandé : «Que peut-on faire quand un lien positif est transformé dans l'interaction, en lien négatif entre deux professeurs? Que faut-il faire ?» Nous sommes alors intervenues en déconstruisant la situation relationnelle entre le Directeur de thèse et son étudiant : d'une part, le doctorant est en position de dominé, au moins quand il commence sa thèse, par rapport à son Directeur de thèse. Mais cette situation peut évoluer au cours de la thèse, pendant laquelle le doctorant va acquérir une expertise, produire des connaissances et tisser le réseau associé à ses activités qui peuvent compléter en partie les ressources spécifiques du Directeur de thèse. Il gagne donc en légitimité, y compris dans celle qui consiste à « imposer » ses relations à son directeur. D'autre part, il est difficile de comprendre la « hiérarchie » des valeurs et des réputations qui fonctionne dans le monde des chercheurs et qui structure aussi l'espace de leurs relations. Ainsi, les réseaux sociaux entre chercheurs n'annihilent pas plus les hiérarchies professionnelles entre eux que les réputations. La difficulté pour le doctorant, qui souhaiterait activer de nouvelles relations, est de comprendre l'existence de ces «hiérarchies » invisibles et d'éviter une activation naïve des chaînes 
relationnelles, à la teneur incertaine. A ce propos, au cours de sa thèse, le doctorant pourra éviter de se trouver enfermé dans le petit réseau de son directeur de thèse et ainsi « casser » la relation dyadique avec lui, pour tisser d'autres contacts et de nouvelles relations et surtout devenir de plus en plus informé et conscient des relations qui n'existent pas entre le directeur, la directrice de thèse et les autres chercheurs du domaine. De ce point de vue, le repérage de l'absence de relations est aussi important que celui des relations existantes.

\section{Quelle chaîne de personnes pour atteindre la cible?}

Le troisième exercice a été mis sur pied en réponse aux attentes des participants à l'atelier, qui faisaient part de difficultés à accéder à des chercheurs ou des universitaires pour des demandes de type conseils, accès au terrain, ou participation à un séminaire.

Il a commencé par ce que nous avons appelé l'exercice «Obama et moi » où MariePierre tente d'imaginer une chaîne activable entre elle et le président des États-Unis, composée de personnes qui seront suffisamment proches et liées par des liens forts, qu'elles devraient faire passer le «message urgent». Il s'agit alors de se rendre compte que les distances relationnelles entre deux personnes qui ne se connaissent pas, peuvent être assez courtes (Milgram) mais aussi que les relations sollicitées ne sont pas prises au hasard dans le carnet d'adresses et qu'il faut réfléchir à la nature des relations entre les différentes personnes sollicitées, afin de maximiser les chances d'atteindre la cible.

A ce moment de l'atelier, il s'agissait de choisir avec chaque doctorant une cible pertinente à atteindre pour conduire sa thèse et de préférence, une cible permettant 
d'accéder à une « ressource » incontournable ou indispensable pour avancer dans le travail doctoral. Chaque cible est ainsi associée à un objectif précis, de type accès à un terrain ou à un matériau, déplacement à l'étranger, obtention d'un financement, prise de contact pour des échanges scientifiques, etc. Afin de susciter plus aisément l'énonciation de cibles potentielles, nous en suggérions deux types: les cibles institutionnelles (Organismes de financement, Universités, Centres de formation, institutions publiques, laboratoires, etc.) et les cibles individuelles et nominatives (chercheur, professeur, personne informée sur le terrain d'investigation, etc.) ou bien plus vraisemblablement la combinaison des deux, à savoir contacter une personne parce qu'elle salariée d'une institution ou bien en contactant un organisme (une équipe de recherche par exemple), rentrer en contact avec un individu dont on n'a pas les coordonnées.

La fable « Obama et moi », suggérait une transposition possible de cet exercice fictif de «tricotage » d'une chaîne dans le contexte de conduite d'une thèse. Le doctorant pourrait atteindre une «ressource» (personne ou organisme) en imaginant une " chaîne » fictive reliant les deux et en passant éventuellement par le réseau, à présent « révélé », du directeur de thèse. L'exercice se déroulait en deux temps : dans un premier temps, les participants avaient pour consigne, à la fin de cette séance d'imaginer pour la prochaine, une « cible » à atteindre et les pistes auxquelles ils avaient pensées, voire qu'ils avaient déjà testées. A la séance suivante, ils justifiaient, devant le groupe, le choix de leur cible, ils nous faisaient part de leurs questions, des chemins imaginés et nous représentions graphiquement au tableau la chaîne relationnelle potentiellement activable, en faisant apparaître les individus, les institutions, et parfois les pays. 
Cette étape s'inspire directement des théories des ressources sociales telles que développées par Nan Lin $(1978,1982,1995)$ qui défend l'idée que les ressources détenues par les relations directes et indirectes des acteurs peuvent les aider à parvenir à leurs fins, que les positions sociales d'origine facilitent l'accès aux ressources sociales et à leur usage et que le choix de relations plus faibles et hétéroclites est performant pour les personnes à statut social élevé. Dans notre cas, la problématique est inverse : le doctorant se situe en bas de l'échelle sociale dans le contexte de réalisation d'une thèse et il doit, en quelque sorte, « remonter la pyramide ». De plus, il ne s'agit pas d'une réelle expérience de sollicitation de relations pour l'accès à des ressources mais d'un exercice fictif.

Sur les 41 étudiants inscrits au départ des clubs successifs, 27 sont allés jusqu'au bout de la formation et ont donc choisi une « cible». Parmi ceux-ci, 15 ont désigné des personnes, dont surtout des chercheurs réputés dans leurs domaines (12 cas) alors que 3 ont choisi d'essayer de contacter des témoins et acteurs de leur «terrain d'investigation ». Parmi les 12 autres qui ont désigné des cibles « institutionnelles », il s'agit essentiellement de recherche de financement (5 cas), et d'institutions universitaires de type organisme de recherche, de formation, d'université ou de bibliothèque, pour les autres.

Sans que cela soit statistiquement significatif, on observe que les étudiants aux «petits réseaux " tenteraient effectivement de contacter plutôt un chercheur; ce qui est également le cas de ceux dotés d'un réseau plus universitaire. La troisième catégorie, qui englobe les doctorants aux réseaux denses et cliqués, n'ont pas porté leur choix sur une 
cible particulière mais se répartissent dans les différentes catégories rencontrées (chercheur, témoin, équipe de recherche, financement, etc.).

La construction des chaînes potentielles fut conduite, à partir de graphes effectués à main levée par les animatrices de la formation et de manière ouverte envers les autres participants. Ceci a souvent permis à l'étudiant concerné d'imaginer plusieurs moyens indirects d'atteindre la cible, comme illustré dans le cas suivant :

Schéma 5 : la fabrication d'une chaîne entre le doctorant et la cible

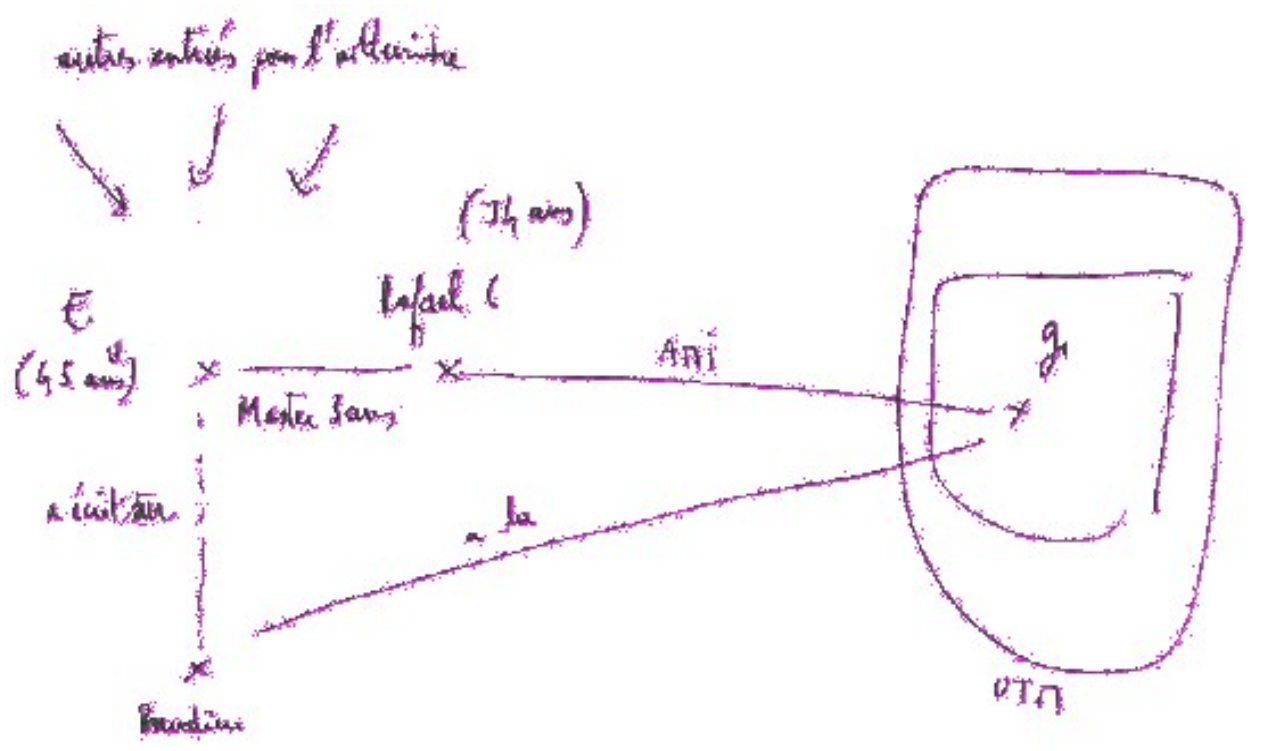

Sur ce dessin d'abord dessiné au tableau puis recopié sur feuille par Marie-Pierre, G. (doctorante), est située à droite et aimerait contacter E., chercheur installé dans un pays étranger, dont elle n'a pas les coordonnées. Elle se souvient qu'elle a un ami (R.) qui a 
fait un Master sous la direction de ce chercheur et que, par son intermédiaire, elle pourrait lui faire passer un message. Elle compte citer, dans sa lettre, le sociologue Pierre Bourdieu car elle sait qu'E. l'apprécie.

Nous pensions que l'activation vraisemblable d'une chaîne de personnes mobilisables pour «aider» un doctorant devait comporter des signaux de qualité, combinant recommandations de personnes (directeur de thèse ou non, autre chercheur, par exemple) et arguments scientifiques de type positionnement théorique, référence à une école de pensée, citation d'autres travaux, etc. Cet aspect nous a conduites pour quelques rares cas, à leur donner des conseils concrets d'élaboration d'une lettre mêlant l'ensemble des ressources repérées précédemment.

Exemple d'un mail de contact entre le doctorant et la cible ${ }^{13}$ :

De : marlene.dietrich@neuf.fr

A : christine.ockrent@noos.fr

Copie à :

Sujet : demande de co-tutelle

\section{Madame le professeur,}

je suis étudiante à l'université Toulouse II le Mirail et je suis titulaire d'un master 2 réalisé sous la direction des professeurs Mme B. et M. N.. Mon mémoire, obtenu avec la mention très bien, portait sur les o... Admise en doctorat, je souhaite continuer à travailler sur $l^{\prime} O$. .

Je me permets de vous solliciter pour vous demander conseil à propos de mon sujet de thèse; j'aimerais interroger la notion d'o....Peut-on encore parler d'o. à cette époque ou doit-on parler de C. ? L'exemple du M. serait pris .... Ce sujet, les bornes chronologiques ainsi que la question posée vous semblent-ils pertinents?

Je sais qu'E.M. travaille, sous votre direction, sur L. et (...). Le sujet que j'envisage est-il trop proche de $13 \quad$ Seuls les noms ont été changés. 
celui de votre étudiante?

Par ailleurs, je souhaiterais bénéficier de vos conseils tout au long de mon doctorat, aussi je vous prie de bien vouloir accepter d'être co-directrice de ma thèse. Vous trouverez ci-joint une copie de mon master 2 qui vous permettras de juger de mon travail.

Salutations distinguées,

Dans ce mail, l'étudiante cite bien ses professeurs directeurs de thèse, elle emploie des termes savants pour parler de sa thèse, et fait référence à une thèse en cours sur un sujet proche au sien puis sollicite enfin une co-tutelle de thèse. Elle recevra d'ailleurs, quelques temps après, une réponse favorable de cette personne.

Mais l'exercice « Obama et moi » comporte des limites évidentes, liées à la question de l'activabilité des liens, et également sur les conseils pédagogiques que les étudiants peuvent retirer de cette formation parfois trop «utilitariste». L'exemple suivant permettra d'illustrer ces points. Eve est une doctorante en fin de thèse, qui a bénéficié d'un financement pour son doctorat, elle est bien insérée dans son laboratoire, mais aussi dans le milieu professionnel dans lequel elle évolue. Elle avait, par exemple, pris l'initiative de monter un dossier de demande $\mathrm{d}^{\prime} \mathrm{ANR}^{14}$, pour son laboratoire et en association avec un organisme extérieur, sur un projet de recherche lié à son terrain d'étude, dans un pays asiatique. Elle souhaitait obtenir une bourse d'un centre de recherche de ce pays pour financer l'accès à son terrain. Il lui paraissait nécessaire d'entrer en contact avec quelqu'un de ce centre de recherche avant de monter le dossier de demande de bourse, pour obtenir plus de renseignements, et savoir comment formuler sa demande. Pendant son récit, Nathalie a dessiné au tableau un schéma intégrant les personnes citées mais aussi les structures dans lesquelles elles évoluent.

$14 \quad$ Projet de recherche financé par l'Agence Nationale de la Recherche. 
Eve s'interrompt alors et s'exclame «c'est marrant mais je viens de me rappeler en regardant ce dessin que l'ancien directeur $d u$ centre de recherche est rattaché à l'organisme extérieur que j'ai cité tout à l'heure et qu'il veut rentrer dans l'ANR ». Eve réfléchit alors, à haute voix, en termes de don/contre-don, se demandant s'il serait possible de d'inciter cette personne à la soutenir auprès de ses anciens collègues pour une demande de financement, en contrepartie de son association à l'ANR. Mais la question suivante est celle de la qualité des relations entre les individus en question. Estil possible et souhaitable d'intégrer un tel, ou tel autre ? Est-ce qu'en le sollicitant, elle ne devient pas son « obligée », selon ses propres termes?

Le cas d'Eve permet d'illustrer deux points fréquemment rencontrés au cours de ces exercices. Premièrement, une grande partie du réseau professionnel des doctorants comme de tout professionnel, est latent. C'est-à-dire que le fait qu'il ne soit pas mentionné au cours du premier exercice ne signifie pas qu'il ne soit pas existant. Deuxième point, les doctorants ne s'autorisent pas nécessairement à contacter toutes les personnes utiles pour leurs thèses, notamment parce qu'ils ont intégré l'idée d'un nécessaire contre-don, dans lequel ils seraient forcément à leur désavantage. Ceci est particulièrement prégnant lorsqu'ils ont le sentiment d'un décalage de position sociale, par exemple entre eux et un chercheur permanent. Cela signifie qu'ils n'ont pas forcément compris l'intérêt que peut avoir, pour un chercheur, le fait d'être en contact avec des doctorants travaillant sur les mêmes thématiques que lui, ne serait-ce qu'en termes d'échanges intellectuels. Ils se sentent en position inégale et dominée, et donc ont l'impression de demander une faveur. Évidemment, se pose également la question de la temporalité : «à quel moment opportun de la thèse un doctorant doit-il contacter un chercheur dans l'optique que ce dernier lui réponde ?» On peut noter ici que ces 
doctorants éprouvent même des difficultés à savoir quoi demander. En effet, un certain nombre d'entre eux ont exprimé l'envie d'entrer en contact avec des chercheurs reconnus sur leurs thématiques mais sans savoir quoi leur dire, sans envisager d'échanges sur un thème précis. Ceux, qui l'ont fait, ont parfois envoyé des courriers de prise de contact sans question réelle et n'ont souvent pas reçu de réponses.

Un autre exemple intéressant, tiré de cet exercice de cible, est celui de Manon, qui voulait contacter une institution de conservation de documents d'un autre pays, avait envoyé en vain des mails à l'adresse apparaissant sur le site internet. A la suggestion de passer par l'intermédiaire d'une institution équivalente en France, avec laquelle elle avait déjà de très bons contacts, pour essayer de savoir, au moins, à qui s'adresser et comment, elle nous a répondu qu'elle n'y avait jamais pensé. Cet exemple illustre une tendance assez fréquente relevée parmi les participants à s'adresser directement à une institution, en fait à un secrétariat anonyme, sans essayer de savoir quelle est la personne ciblée, afin de l'atteindre directement. Ce mode de fonctionnement débouche souvent sur des non réponses, le découragement s'installe, et les étudiants renoncent à d'autres démarches, les considérant vaines par avance. L'idée de demander à des collègues de travail de leur laboratoire, ou de toute autre institution dans laquelle ils évoluent, d'éventuels conseils, suggestions ou mises en contact, ne leur paraît pas envisageable.

Il est remarquable de noter aussi que les doctorants ont rarement mentionné leurs directeurs de thèse ou même les membres de leur laboratoire dans les chaînes relationnelles susceptibles de les conduire à une ressource. L'idée de tenir au courant le directeur de thèse des démarches voire de lui demander d'être un intermédiaire est apparue évidente après coup, mais pas sur le moment. Ceci rejoint l'impression d'une 
solitude du doctorant sur son sujet, d'un isolement souvent exprimé par des expressions de type : "personne ne travaille sur le même sujet que moi, je ne vois pas qui je pourrais rencontrer $\gg$. L'exercice de la cible a finalement eu surtout comme résultat de permettre de prendre conscience d'un réseau professionnel potentiel beaucoup plus large qu'ils ne se l'imaginaient, en y associant les personnes ou institutions ayant des points ou sujets communs avec chaque doctorant et son entourage d'étude. Les dernières discussions ont porté sur la visibilisation de la chaîne activée envers les participants : un doctorant qui écrit à un chercheur confirmé doit-il se recommander de son directeur de thèse ? doit-il aussi avertir le directeur de thèse qu'il le fait ? de manière simultanée ? avec un décalage dans le temps?

\section{Conclusion :}

Cette recherche-action permet d'avancer un certain nombre de propositions sur la nature latente des relations et des structures relationnelles associées, réseau et chaîne :

A un premier niveau, celui des relations, on avancera qu'une relation latente est une relation potentielle d'un individu qui pourrait le relier directement à un autre. Elle n'est que latente parce qu'il n'y pense pas ou bien parce qu'il n'ose pas l'activer. C'est le cas pour les doctorants, qui oublient qu'ils connaissent certains chercheurs déjà rencontrés ou sollicités ou bien n'osent pas les contacter.

A un deuxième niveau, celui du réseau personnel latent, il s'agit d'une partie du réseau personnel, composé des relations directes non activées et des relations indirectes effectives et positives. Si les premières étaient activées, il pourrait facilement atteindre les secondes. Plus l'on s'éloigne d'Ego, plus la part du réseau latent est forte sur le réseau personnel connu et décrit par lui. C'est le cas de doctorants qui ne connaissent 
pas le réseau de leurs directeurs de thèse ou encadrants et qui pèchent plutôt par leur méconnaissance des relations indirectes de niveau 1 et 2 .

Une chaîne latente est un chemin relationnel entre plusieurs personnes, composé de plusieurs relations dyadiques plus ou moins activables par une série de recommandations, à établir en fonction de la ressource sociale considérée, mais dont il nécessaire de connaître, à chaque étape, la nature (intensité, fréquence de contacts, etc.), la réciprocité (nature de l'engagement, de la confiance) et les « coutumes » d'activation dans les différents cercles sociaux traversés. Elle n'est donc qu'une suite potentielle de relations dyadiques, trop dissemblables pour former une chaîne relationnelle véritable et spécifique à la ressource sociale visée. Son activation réussie nécessite une série de recommandations et de signaux de reconnaissance difficiles à mélanger et à combiner opportunément pour atteindre la ressource visée. Ainsi, le doctorant qui cherche à photographier un tableau rare n'active pas la même chaîne relationnelle ni de la même façon que celui qui cherche un co-encadrement de thèse. 


\section{références bibliographiques :}

Bès Marie-Pierre, Chaulet Johann, 2012 «Le travail relationnel des Associations d'anciens élèves dans la reproduction du capital social et la distinction entre élites », Communication à la Journée «Faire de la sociologie économique avec Pierre Bourdieu », Université Lille 1.

Bès Marie-Pierre, 2004 «Connaissances et relations sociales des jeunes chercheurs », Recherches Sociologiques, vol. 3, pp. 123-135.

Bès Marie-Pierre et Chauvac Nathalie, 2011 «Les réseaux des doctorants : Le cas d'étudiants en Sciences Humaines de l'Université de Toulouse Le Mirail », Présentation aux Journées de l'Observation de la Vie Étudiante, Toulouse.

Bidart Claire, Degenne Alain \& Grossetti Michel, 2011 «la vie en réseau : dynamique des relations sociales », Presses Universitaires de France,.

Biron, Jean-François, Julie Montreuil, Johanne Saint-Charles, Pierre Mongeau, et Serge Chevalier, 2008 «Profil de l'insertion sociale par l'analyse de réseau : un outil pour la relation d'aide ». (http://collections.banq.qc.ca/ark:/52327/bs65891).

Granovetter Mark, 1974 Getting a job: a Study of Contacts and Careers. Cambridge, Mass., Harvard University Press.

Godechot Olivier, Louvet Alexandra, 2010, «Comment les docteurs deviennent-ils directeurs de thèse ? Le rôle des réseaux disponibles », Sociologie, vol. 1, n 1, 3-23.

Godechot Olivier, Mariot Nicolas, 2004, « Les deux formes du capital social. Structure relationnelle des jurys de thèse et recrutement en science politique $»$, Revue française 
de sociologie, vol. $45, \mathrm{n}^{\circ} 2$, p. 243-282.

Lazuech Gilles, 2000, « Recruter, être recrutable. L'insertion professionnelle des jeunes diplômés d'écoles d'ingénieurs et de commerce », Formation emploi n 69, pp. 5-19.

Lecoutre Marc, 2006 ,Le capital social dans les transitions entre l'école et l'entreprise, La Découverte/M.A.U.S.S..

Lin Nan, 1995 «Les ressources sociales : une théorie du capital social », Revue française de sociologie,, Volume 36, Numéro 36-4, pp. 685-704.

Lin Nan, Dayton Paul W., and Greenwald Peter, 1978 “Analyzing the Instrumental Use of Relations in the Context of Social Structure", Sociological Methods \& Research, $\mathrm{n}^{\circ} 7$, p. $149-166$.

Milgram Stanley, "The Small World Problem", Psychology Today, Vol. 2, 60-67, 1967.

Milgram Stanley \& Travers Jeffrey, 1969« An Experimental Study of the Small World Problem », Sociometry, Vol. 32, No. 4. (1), pp. 425-443.

Shinn Terry, 1988 « Hiérarchies des chercheurs et formes des recherches », Actes de la recherche en sciences sociales, $\mathrm{n}^{\circ} 74$, pp. 2-22. 